OPEN ACCESS

Edited by:

Feng Liu,

Tianjin Medical University General Hospital, China

Reviewed by:

Junfeng Sun,

Shanghai Jiao Tong University, China

Daifa Wang,

Beihang University, China

Qi Han Zhang,

Tianjin Normal University, China

*Correspondence:

Kuo Zhang

zhangkuo1006@tju.edu.cn

Dong Ming

richardming@tju.edu.cn

Specialty section:

This article was submitted to

Brain Imaging Methods,

a section of the journal

Frontiers in Neuroscience

Received: 11 April 2021

Accepted: 07 June 2021

Published: 16 August 2021

Citation:

Si X, Xiang S, Zhang L, Li S,

Zhang K and Ming D (2021)

Acupuncture With deqi Modulates

the Hemodynamic Response and Functional Connectivity of the Prefrontal-Motor Cortical Network.

Front. Neurosci. 15:693623. doi: 10.3389/fnins.2021.693623

\section{Acupuncture With deqi Modulates the Hemodynamic Response and Functional Connectivity of the Prefrontal-Motor Cortical Network}

\author{
Xiaopeng Si $i^{1,2,3,4}$, Shaoxin Xiang ${ }^{2,3}$, Ludan Zhang ${ }^{1,2}$, Sicheng Li $^{1,2}$, Kuo Zhang ${ }^{1,2 *}$ and \\ Dong Ming ${ }^{1,2 *}$
}

'Academy of Medical Engineering and Translational Medicine, Tianjin University, Tianjin, China, ${ }^{2}$ Tianjin Key Laboratory of Brain Science and Neural Engineering, Tianjin University, Tianjin, China, ${ }^{3}$ Tianjin International Engineering Institute, Tianjin University, Tianjin, China, ${ }^{4}$ Institute of Applied Psychology, Tianjin University, Tianjin, China

As a world intangible cultural heritage, acupuncture is considered an essential modality of complementary and alternative therapy to Western medicine. Despite acupuncture's long history and public acceptance, how the cortical network is modulated by acupuncture remains largely unclear. Moreover, as the basic acupuncture unit for regulating the central nervous system, how the cortical network is modulated during acupuncture at the Hegu acupoint is mostly unclear. Here, multi-channel functional near-infrared spectroscopy (fNIRS) data were recorded from twenty healthy subjects for acupuncture manipulation, pre- and post-manipulation tactile controls, and preand post-acupuncture rest controls. Results showed that: (1) acupuncture manipulation caused significantly increased acupuncture behavioral deqi performance compared with tactile controls. (2) The bilateral prefrontal cortex (PFC) and motor cortex were significantly inhibited during acupuncture manipulation than controls, which was evidenced by the decreased power of oxygenated hemoglobin $(\mathrm{HbO})$ concentration. (3) The bilateral PFC's hemodynamic responses showed a positive correlation trend with acupuncture behavioral performance. (4) The network connections with bilateral PFC as nodes showed significantly increased functional connectivity during acupuncture manipulation compared with controls. (5) Meanwhile, the network's efficiency was improved by acupuncture manipulation, evidenced by the increased global efficiency and decreased shortest path length. Taken together, these results reveal that a cooperative PFC-Motor functional network could be modulated by acupuncture manipulation at the Hegu acupoint. This study provides neuroimaging evidence that explains acupuncture's neuromodulation effects on the cortical network.

Keywords: acupuncture, functional connectivity, functional near-infrared spectroscopy, motor cortex, prefrontal cortex

\section{INTRODUCTION}

External neurostimulation and its modulatory effect on the cortical network have been the subject of extensive research by neuroscientists. As an external neuromodulation method that originated in ancient China, acupuncture balances sympathetic and parasympathetic nervous activity (Takahashi, 2011) and modulates the human brain (Yu et al., 2018). As a world intangible 
cultural heritage, acupuncture is now considered an essential mode of complementary and alternative therapy to Western medicine (Yoo et al., 2004). Studies have demonstrated that acupuncture has therapeutic effects on various neuropsychiatric disorders such as neuropathic pains (Miranda et al., 2015), Alzheimer's disease (Liang et al., 2014), and Parkinson's disease (Jiang et al., 2018). Besides acupuncture's clinical therapeutic effects, it has been shown that acupuncture could enhance human cognition and memory (Zheng et al., 2018) and regulate emotion processing for healthy people (Hui et al., 2005). Despite acupuncture's long history and public acceptance, the underlying neural mechanism of acupuncture's neuromodulation effects on the cerebral cortex remain unclear.

The needling sensation that subjects experience during acupuncture is called deqi (Hui et al., 2007), which refers to the excitation of vital energy, known as $q i$ in languages from China (Kong et al., 2007). Importantly, deqi is an indispensable requirement for achieving acupuncture efficacy according to Traditional Chinese Medicine (TCM) (Sun et al., 2020). Previous studies showed that acupuncture with deqi sensation could produce better therapeutic efficacy and reduce the severity of symptoms compared to no deqi sensation (Hui et al., 2007; Fang et al., 2009; Lee et al., 2017). Therefore, deqi was employed as a valuable behavioral index to evaluate the acupuncture effect (Yang et al., 2013). However, the current behavioral evaluation of deqi is subjective and insufficient. There is a lack of an objective neural biomarker for quantifying acupuncture's deqi sensation.

The human brain is an integrative and complex network system (Bassett and Sporns, 2017; Cai et al., 2018), in which regions are considered as nodes, and functional or structural connectivity between regions is considered as edges (van den Heuvel and Pol, 2010). According to the network control theory in network neuroscience, the brain's functional network could be modulated by various neuromodulation methods, such as acupuncture (Takahashi, 2011), transcranial magnetic stimulation (TMS) (Curtin et al., 2019b), transcranial electrical stimulation (tES) (Wang et al., 2019), and transcranial focused ultrasound (tFUS) (Legon et al., 2014).

During the acupuncture stimulation process, the afferent neural activities from the peripheral nervous system are firstly carried up to the brainstem and then are transferred to the subcortical regions in the central nervous system, such as the thalamus, cerebellum, amygdala (Zhang et al., 2012). Finally, the high-level cerebral cortex of the human brain, such as the prefrontal and sensorimotor network, is modulated by the afferent signals from subcortical regions (Lund and Lundeberg, 2016). By modulating the cortical network during acupuncture stimulation, the human brain's cognition functions and behavioral outcomes are finally regulated (Zheng et al., 2018; Ghafoor et al., 2019). However, how the brain's functional cortical network changes and what specific cortical regions are involved during acupuncture with deqi remain mostly unclear.

To reveal how the human brain's functional network changes during acupuncture with deqi, different neuroimaging methods, such as functional magnetic resonance imaging (fMRI) and electroencephalography (EEG) have been used in previous studies. The high-spatial-resolution fMRI studies mainly revealed the involvement of several subcortical brain regions during acupuncture (Hui et al., 2005; Dhond et al., 2008; Fang et al., 2009; Asghar et al., 2010; Hui et al., 2010; Zhong et al., 2012). For example, it was found that the limbic-paralimbicneocortical network, including the subgenual anterior cingulate cortex, posterior cingulate cortex, amygdala, and hippocampus, was deactivated during acupuncture (Dhond et al., 2008; Fang et al., 2009; Zhong et al., 2012), whereas the insula, thalamus, the somatosensory, and anterior cingulate cortex were activated by acupuncture (Dhond et al., 2008; Zhong et al., 2012). Besides, fMRI studies also revealed that the sub-cortical regions' functional connectivity could be modulated during acupuncture (Dhond et al., 2008; Zhong et al., 2012). Previous fMRI studies have found that changes in the sub-cortical regions' functional connectivity during acupuncture, but only a few studies focused on the cortical network changes, but how the cortical network is modulated during acupuncture with deqi remains unclear.

Meanwhile, scalp EEG was also used to explore the modulation effect of acupuncture, which found that the delta and theta frequency band's EEG power (Yu et al., 2017, 2018) and functional connectivity were increased by acupuncture stimulation (Chen et al., 2006; Yu et al., 2017, 2019). However, due to the volume conduction effect (Hipp et al., 2012) and the low-spatial resolution (Tabar and Halici, 2017), EEG failed to reveal the specific cortical region's involvement during acupuncture. In summary, how the key node and functional connectivity of the cortical network are modulated by acupuncture with deqi remains mostly unclear.

The functional near-infrared spectroscopy (fNIRS), a relatively novel non-invasive neuroimaging technique (Quaresima and Ferrari, 2019), is good at detecting the cerebral cortex's hemodynamic responses, such as oxygenated hemoglobin (HbO) and deoxygenated hemoglobin (HbR) changes, which could reflect the underlying cortical neural activities according to the neurovascular coupling effect (Keles et al., 2016; Soltanlou et al., 2018). Compared with EEG, fNIRS has a much higher spatial-resolution and is especially good for detecting cortical neural activities (Wang et al., 2020). Moreover, compared to fMRI, fNIRS is both portable and less sensitive to motion artifacts. As a useful neuroimaging tool, fNIRS is widely used to investigate the high-level cerebral cortex's hemodynamic activities and the related functional network in various neuromodulation studies, such as TMS (Park et al., 2017; Curtin et al., 2019b), tES (Patel et al., 2020), and acupuncture (Takamoto et al., 2010; Ghafoor et al., 2019; Rojas et al., 2019). Previous acupuncture studies also showed the prospect of applying fNIRS to study acupuncture's neural mechanism (Takamoto et al., 2010; Ghafoor et al., 2019; Rojas et al., 2019). However, to our knowledge, few studies used fNIRS to simultaneously investigate the cortical response and cortico-cortical functional connectivity during acupuncture with deqi.

The Hegu acupoint (LI-4) is considered the basic neural acupuncture unit (Zhang et al., 2012) for regulating the central nervous system according to the TCM. For example, acupuncturing at the Hegu acupoint with deqi could relieve pain (Liang et al., 2014) and improve the human brain's cognition 
(Zheng et al., 2018) by balancing the brain activity as a whole. In addition, previous studies have shown that acupuncture at the Hegu acupoint could regulate the activity of the brain, activating or inhibiting areas of the brain to achieve the effect of anesthetic (Hui et al., 2010; Liang et al., 2014). However, how the cortical network is modulated during acupuncture at Hegu acupoint is largely unclear. Considering that the human brain is an integrative and complex network system (Bassett and Sporns, 2017; Cai et al., 2018), we hypothesized that acupuncture at the Hegu acupoint with deqi could achieve its acupuncture effects by modulating the large-scale cortical network. The functional cortical network revealed by fNIRS could thus be used as an objective biomarker to quantify the behavior deqi effect for acupuncture.

To test our hypothesis, the multi-channel fNIRS data were recorded from twenty healthy subjects for acupuncture manipulation, pre- and post-manipulation tactile controls, and pre- and post-acupuncture rest controls. Meanwhile, each subject's acupuncture deqi behavior performance was collected. As the basic acupuncture unit for regulating the central nervous system according to TCM (Zhang et al., 2012), the Hegu acupoint was employed in this study to explore the neuromodulation effects of acupuncture. To reveal the acupuncture with deqi responsive areas, the hemodynamic responses and the power of $\mathrm{HbO}$ concentration were computed and compared between different conditions. To investigate the correlation relationship between hemodynamic response and behavior performance for acupuncture with deqi responsive areas, Pearson's correlation was analyzed. To reveal how the cortical network is modulated by acupuncture with deqi, the functional connectivity and graph theory parameters of the network were calculated and compared between acupuncture manipulation and controls.

\section{MATERIALS AND METHODS}

\section{Participants}

Twenty healthy right-handed volunteers $(23.9 \pm 1.5$ years old (mean \pm std), age range from 22 to 28 years, twelve males and eight females) were recruited for the acupuncture experiment (Table 1). All subjects have normal intelligence, no history of mental illness, and no brain damage caused by long-term medication. Most importantly, all subjects in this study were naive to acupuncture, having never experienced an acupuncture treatment. After confirming that each subject met all the inclusion criteria, subjects gave written informed consent prior to participating, which was reviewed by the Institutional Review Board and Ethics Committee of Tianjin University.

\section{Acupuncture Experiment Paradigm}

The acupuncture manipulation was performed by an experienced acupuncturist. The acupuncture site was the Hegu acupoint, also known as "Large intestine 4" (LI4) at the midpoint on the radial side of the second metacarpal (Kong et al., 2015; Figure 1). Throughout the experiment, all subjects were asked to sit in a comfortable chair during the entire process of the acupuncture experiment in a quiet room (Figure 2A). All subjects
TABLE 1 | Detailed information of subjects.

\begin{tabular}{|c|c|c|c|c|}
\hline Subject & $\begin{array}{c}\text { Age } \\
\text { (years) }\end{array}$ & Gender & $\begin{array}{c}\text { Accepted } \\
\text { channels/All }\end{array}$ & $\begin{array}{c}\text { Accepted } \\
\text { (>50\% channels } \\
\text { accepted) }\end{array}$ \\
\hline S1 & 28 & Male & $35 / 48$ & Yes \\
\hline $\mathrm{S} 2$ & 23 & Male & $35 / 48$ & Yes \\
\hline S3 & 23 & Female & $30 / 48$ & Yes \\
\hline S4 & 25 & Male & $46 / 48$ & Yes \\
\hline S5 & 24 & Male & $33 / 48$ & Yes \\
\hline S6 & 23 & Female & $35 / 48$ & Yes \\
\hline S7 & 22 & Male & $47 / 48$ & Yes \\
\hline S8 & 23 & Male & $34 / 48$ & Yes \\
\hline S9 & 27 & Male & $46 / 48$ & Yes \\
\hline $\mathrm{S} 10$ & 23 & Female & $34 / 48$ & Yes \\
\hline S11 & 24 & Male & $46 / 48$ & Yes \\
\hline $\mathrm{S} 12$ & 22 & Male & $38 / 48$ & Yes \\
\hline $\mathrm{S} 13$ & 23 & Male & $25 / 48$ & Yes \\
\hline $\mathrm{S} 14$ & 25 & Male & $22 / 48$ & No \\
\hline S15 & 24 & Female & $47 / 48$ & Yes \\
\hline S16 & 23 & Female & $34 / 48$ & Yes \\
\hline $\mathrm{S} 17$ & 24 & Male & $34 / 48$ & Yes \\
\hline $\mathrm{S} 18$ & 23 & Female & $15 / 48$ & No \\
\hline S19 & 23 & Female & $46 / 48$ & Yes \\
\hline $\mathrm{S} 20$ & 26 & Female & $13 / 48$ & No \\
\hline
\end{tabular}

were required to keep relaxed and to keep their heads still during the experiment.

During the preparation, each subject was asked to familiarize themself with the deqi sensation behavior questionnaire, containing six individual visual analog scales (VAS) for evaluating the multiple acupuncture deqi sensations (Kou et al., 2007), which including suan (aching or soreness), ma (numbness or tingling), zhang (fullness/distention or pressure), tong (dull pain), zouchuan (spreading, a feeling of extension from the acupuncture position to the arm) and zhong (heaviness) according to TCM (Asghar et al., 2010; Hui et al., 2011; Yu et al., 2012). The intensity of each needing sensation was rated by a point between 0 (none) and 10 (strongest) to quantify each of the six sensations for each subject.

Considering that the quasi-experimental design could involve the real-world acupuncture intervention instead of artificial experimental settings with high external validity (Bärnighausen et al., 2017), the quasi-experimental design was employed in this study (Figure 1), which was also used in previous acupuncture studies (Yu et al., 2017; Yu et al., 2018). The whole acupuncture procedure included the following five conditions (Figure 1). (I) Pre-acupuncture rest control: each subject was asked to keep awake and rest for 5 min without movement before the needle was inserted by the acupuncturist. (II) Pre-manipulation tactile control: a single-use sterile acupuncture needle was inserted by the acupuncturist at the Hegu acupoint to a certain depth without twisting. To avoid the bias of acupuncture site, random acupuncture on the right or left hand at the Hegu acupoint was conducted in this experiment. During this condition, each subject was asked to maintain awake and rest for 5 min before 


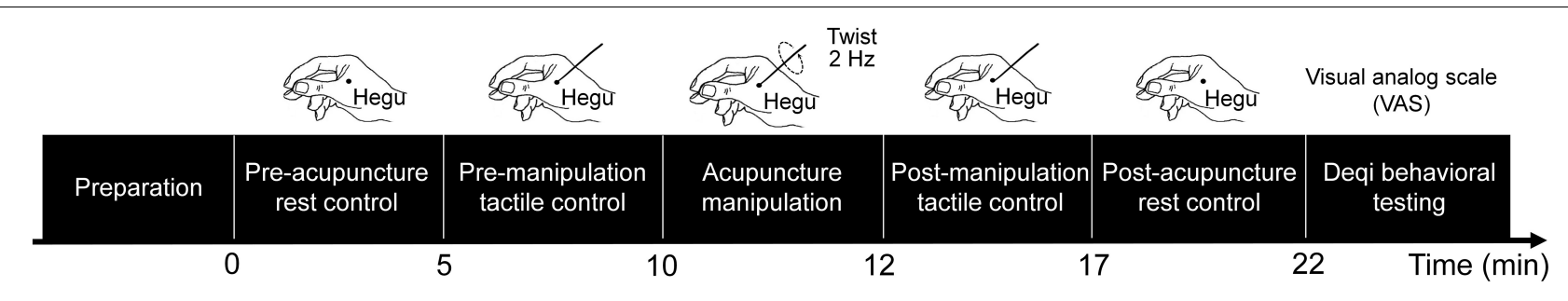

FIGURE 1 | Acupuncture experiment paradigm. During the preparation, subjects were asked to be familiar with the deqi behavioral questionnaire evaluated by the visual analog scale (VAS). There are five conditions in the experiment: pre-acupuncture rest control (5 min), pre-manipulation tactile control (5 min), acupuncture manipulation (2 min), post-manipulation tactile control (5 min), and post-acupuncture rest control (5 min). After the experiment, subjects were asked to evaluate the needling sensations for the pre-manipulation tactile control, acupuncture manipulation, and post-manipulation tactile control. The twisting frequency in acupuncture manipulation is $2 \mathrm{~Hz}$.

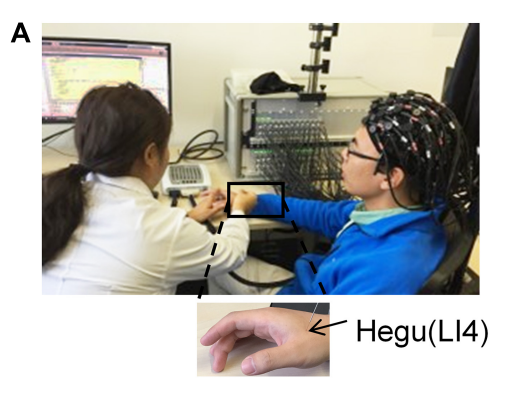

c

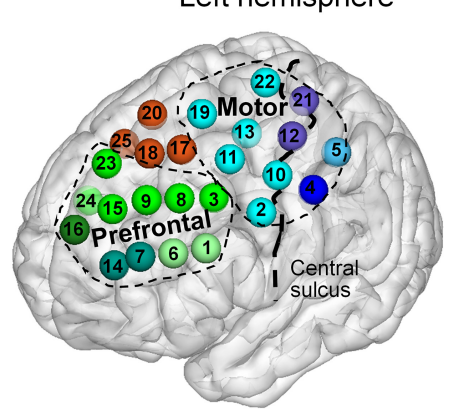

B

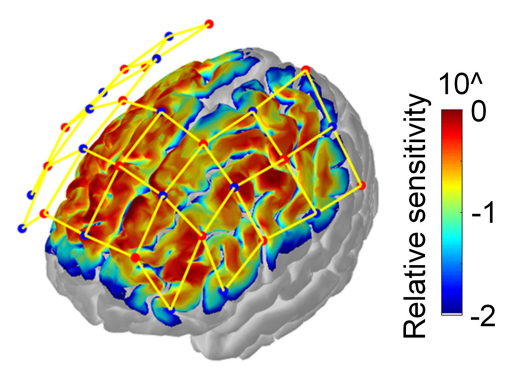

Right hemisphere
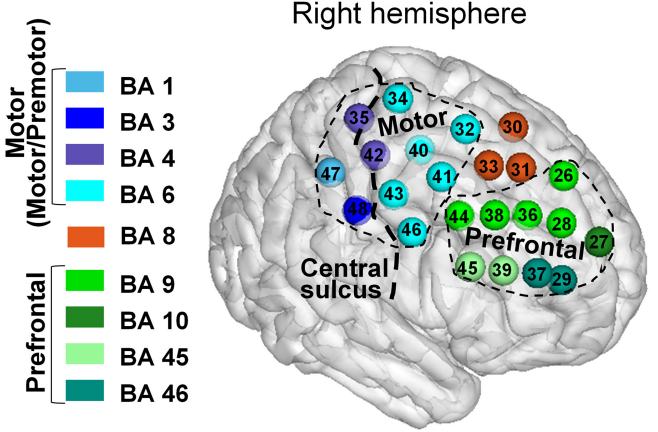

FIGURE 2 | Acupuncture experiment, fNIRS channel configuration, and anatomical location. (A) Acupuncture experiment. An experienced acupuncturist conducted acupuncture on the subject wearing a 48 channel fNIRS measuring cap. The black arrow indicated the location of the Hegu acupoint (LI-4). (B) fNIRS channel configuration. Red dots represented light sources, and blue dots represented detectors. Each fNIRS channel's relative cortical sensitivity was visualized by pseudo-color. (C) Anatomical location. Each channel's Brodmann label was indicated by a different color. All channels were divided into three regions of interests (ROIs), the prefrontal cortex (PFC, colored with the green series system), the motor-related area (motor and premotor cortex, colored with the blue series system), and the remaining Brodmann area 8 (BA 8, colored with red, BA, Brodmann area).

the acupuncture twisting. (III) Acupuncture manipulation: the subjects were acupunctured by using the twirling method at the Hegu acupoint for $2 \mathrm{~min}$. The twisting is mainly manipulated by the thumb to push forward with force, which was within a range of $90^{\circ}-180^{\circ}$ ( $\mathrm{Yu}$ et al., 2017) with a frequency of $2 \mathrm{~Hz}$. (IV) Post-manipulation tactile control: after the acupuncture manipulation period, each subject was asked to keep awake in a resting state with a needle inserted for $5 \mathrm{~min}$. During this condition, the needle was kept at the Hegu acupoint without the twist. (V) Post-acupuncture rest control: after the acupuncturist removed the needle, each subject was asked to maintain awake and rest for $5 \mathrm{~min}$ without movement. Immediately after the acupuncture experiment, the behavior questionnaire was given to each subject to evaluate his/her deqi sensation for the following three conditions: pre-manipulation tactile control, acupuncture manipulation, post-manipulation tactile control.

\section{fNIRS Data Acquisition}

The fNIRS measurements were conducted by a multichannel fNIRS system (NirScan, Danyang Huichuang Medical Equipment Co., Ltd.) at a sampling rate of $9 \mathrm{~Hz}$. Near-infrared light of three different wavelengths $(740,808$, and $850 \mathrm{~nm})$ was used to detect the concentration signals of $\mathrm{HbO}$ and $\mathrm{HbR}$. For the channel configuration, 15 light sources and 16 detectors were plugged into a holder and resulted in 48 measurement channels, 
which were positioned by referring to the standard international 10-20 system of electrode placement (Figure 2B). The distance between the light source and the detectors was $3 \mathrm{~cm}$.

\section{fNIRS Channel Location and Cortical Sensitivity}

To obtain the Montreal Neurological Institute (MNI) coordinates of each fNIRS channel, the spatial coordinates of the light sources, detectors, and anchor points (located at $\mathrm{Nz}, \mathrm{Cz}, \mathrm{Al}, \mathrm{Ar}, \mathrm{Iz}$ referring to the standard international 10-20 system of electrode placement) were firstly measured by using the electromagnetic 3D digitizer system (FASTRAK. Polhemus VT, United States). Then, the spatial coordinates of the light sources and detectors were registered to the standard MNI space by using the spatial registration approach (Tsuzuki et al., 2007). Thirdly, the MNI coordinate of the midpoint between a light source and detector pair was determined, as each fNIRS channel consisted of a light source and detector pair. Fourthly, the midpoint was spatially projected to the cortex surface of the MNI "Colin27" brain template, and the fNIRS channel's coordinate was defined as the MNI coordinate of the cortical projected point. Finally, to reduce the measurement error, the coordinates of left/right hemispheric symmetric channels were averaged and marked with positive or negative hemispheric information (Table 2). To reveal the probability of the transmitted photon path in the cortex, MonteCarlo photon transmitted software tMCimg was used to obtain the cortical sensitivity (Boas et al., 2002). Then the cortical sensitivity of each channel was displayed on the MNI "Colin27" brain template by using the AtlasViewer (Aasted et al., 2015; Figure 2B). The cortical sensitivity information of each channel was then used to visualize different indexes, such as statistical significance, hemoglobin concentration, and channel label.

\section{Brodmann Atlas (BA) Label and Regions of Interest (ROI)}

To obtain the BA label of each channel, the MNI-to-BA Tool (Okamoto et al., 2009) was used. Firstly, a $10 \mathrm{~mm}$ radius sphere with each fNIRS channel's MNI coordinate as the center was determined. Secondly, all possible BA labels within the sphere were determined. Then, the percentage of each BA label's voxel number within the sphere over the entire sphere's voxel number was calculated. Thirdly, the BA label with the highest percentage was identified as the BA label of a given fNIRS channel. Finally, each fNIRS channel and the corresponding BA label were visualized on the "Colin27" brain template by using BrainNet Viewer (Xia et al., 2013; Figure 2C). According to each fNIRS channel's BA label and the anatomical location information from the "Colin27" brain template, all fNIRS channels were divided into the following three ROIs, including the motor area, the prefrontal cortex (PFC) area, and the remaining Brodmann area 8 (Figure 2C).

\section{The Acupuncture's Behavioral deqi Index Calculation and Statistical Analysis}

To quantify the acupuncture's deqi behavioral effect, the Massachusetts general hospital acupuncture sensation scale
TABLE 2 | The MNI coordination and Brodmann label of each channel.

\begin{tabular}{|c|c|c|c|c|c|}
\hline Channel & $x$ & $\mathbf{Y}$ & $\mathbf{z}$ & Brodmann label & ROI \\
\hline Ch1/Ch45 & $-54 / 54$ & 31 & 27 & BA 45 & Prefrontal \\
\hline Ch2/Ch46 & $-59 / 59$ & 8 & 39 & BA 6 & Motor \\
\hline Ch3/Ch44 & $-46 / 46$ & 22 & 44 & BA 9 & Prefrontal \\
\hline Ch4/Ch48 & $-60 / 60$ & -16 & 44 & BA 3 & Motor \\
\hline Ch5/Ch47 & $-54 / 54$ & -31 & 55 & $\mathrm{BA} 1$ & Motor \\
\hline Ch6/Ch39 & $-47 / 47$ & 42 & 25 & BA 45 & Prefrontal \\
\hline Ch7/Ch37 & $-43 / 43$ & 55 & 25 & BA 46 & Prefrontal \\
\hline Ch8/Ch38 & $-41 / 41$ & 35 & 45 & BA 9 & Prefrontal \\
\hline Ch9/Ch36 & $-31 / 31$ & 44 & 43 & BA 9 & Prefrontal \\
\hline Ch10/Ch43 & $-54 / 54$ & -3 & 51 & BA 6 & Motor \\
\hline Ch11/Ch41 & $-44 / 44$ & 13 & 58 & BA 6 & Motor \\
\hline Ch12/Ch42 & $-45 / 45$ & -16 & 62 & BA 4 & Motor \\
\hline Ch13/Ch40 & $-32 / 32$ & -3 & 62 & BA 6 & Motor \\
\hline Ch14/Ch29 & $-32 / 32$ & 60 & 19 & BA 46 & Prefrontal \\
\hline Ch15/Ch28 & $-23 / 23$ & 55 & 39 & BA 9 & Prefrontal \\
\hline Ch16/Ch27 & $-14 / 14$ & 67 & 30 & BA 10 & Prefrontal \\
\hline Ch17/Ch33 & $-29 / 29$ & 26 & 60 & BA 8 & BA8 \\
\hline Ch18/Ch31 & $-21 / 21$ & 36 & 58 & BA 8 & BA8 \\
\hline Ch19/Ch32 & $-24 / 24$ & 13 & 71 & BA 6 & Motor \\
\hline Ch20/Ch30 & $-5 / 5$ & 24 & 67 & BA 8 & BA8 \\
\hline Ch21/Ch35 & $-34 / 34$ & -29 & 71 & BA 4 & Motor \\
\hline Ch22/Ch34 & $-26 / 26$ & -16 & 79 & BA 6 & Motor \\
\hline Ch23/Ch26 & $-10 / 10$ & 49 & 53 & BA 9 & Prefrontal \\
\hline Ch24 & 2 & 50 & 34 & BA 9 & Prefrontal \\
\hline Ch25 & 3 & 32 & 54 & BA 8 & BA8 \\
\hline
\end{tabular}

$B A$, Brodmann area, $R O I$, region of interest.

(MASS) was administered as the deqi index to quantify the needling sensation in this study (Kong et al., 2007; Yu et al., 2012). According to the MASS index, the VAS scores for all the different needling sensations were sorted from highest to lowest for each subject. The deqi index was calculated according to the following equation:

$$
\text { deqi index }=\frac{\sum_{i=1}^{n}(1 / 2)^{i} R_{i}}{1-(1 / 2)^{n}},
$$

where $R_{i}$ indicates the highest to lowest ranking index of $i$ th VAS score, and $n$ represents the number of different deqi sensations on the behavior questionnaire. The deqi index was calculated for each subject (Supplementary Table 1).

To evaluate if there is a significant deqi sensation difference between different conditions, paired $t$-tests were used on all subjects' deqi data for tactile controls (averaging two tactile controls) vs. acupuncture manipulation (Figure 2A), and for premanipulation tactile control vs. post-manipulation tactile control (Figure 3), respectively. The $p$-values were corrected with false discovery rate (FDR) correction for multiple comparisons to minimize the risk of type I errors. To verify the validity of the statistical results, the statistical power was also calculated by using the free software $G^{*}$ power.

\section{fNIRS Data Pre-processing}

Because the fNIRS recorded raw data contained various noises, fNIRS raw data was pre-processed with the MATLAB ${ }^{\mathrm{TM}} 17.0$ 


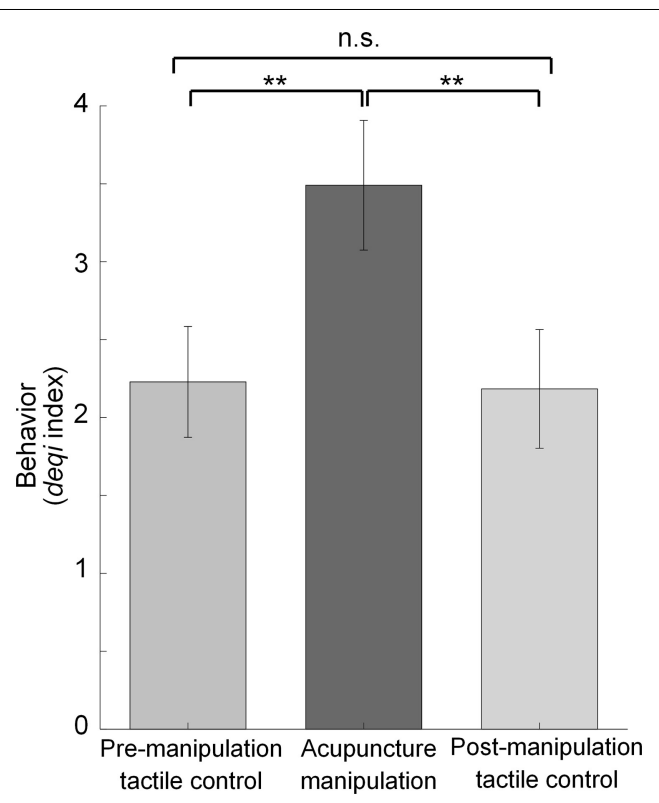

FIGURE 3 | Statistical comparisons of behavior deqi index for different conditions. Behavior results comparison for pre-manipulation tactile control vs. acupuncture manipulation vs. post-manipulation tactile control. (mean \pm SEM, paired $t$-tests with FDR correction for multiple comparisons, $n=17,{ }^{\star \star} p<0.01$ and statistical power $>0.95$, n.s. for non-significance).

(MathWorks, United States) software by the following procedure to improve the signal-to-noise ratio (SNR) (Supplementary Figure 1). Firstly, noisy channels were rejected if any of the following situations were met: (I) The optical intensity (OI) range exceeded the $0.5-1,000$; (II) The source-detector separation range exceeded the range from 0 to 45 ; and (III) The ratio of the mean and standard deviation of the optical intensity [mean(OI)/std(OI)] is less than 2 (Huppert et al., 2009; Peters et al., 2020). Secondly, for a given subject, if the percentage of the subject's rejected channels over all channels exceeds 50\%, the subject with poor signal quality was also rejected, which was in line with the previous fNIRS study (Hakuno et al., 2020). In total, there were three subjects (S14, S18, S20) excluded from the subsequent analysis (Table 1). Thirdly, after excluding the noisy channels and subjects, the optical intensity raw data were converted into optical density (OD) (Supplementary Figure 1A).

To further improve the SNR of $\mathrm{HbO}$ and $\mathrm{HbR}$, the following four pre-processing steps were applied (Supplementary Figure 1). In step 1, motion artifacts containing in the OD signals were corrected by using principal component analysis (PCA) (Supplementary Figure 1B; Behrendt et al., 2018; Di Lorenzo et al., 2019). In step 2, to remove various physiological noises such as cardiac $(\sim 1 \mathrm{~Hz})$, respiration $(\sim 0.3 \mathrm{~Hz})$, and low-frequency drifts $(<0.09 \mathrm{~Hz})$, the $\mathrm{OD}$ data were filtered by a $0.01-0.2 \mathrm{~Hz}$ Butterworth bandpass filter (Scholkmann et al., 2014; Chen and Glover, 2015; Pinti et al., 2019), which is in line with previous fNIRS studies (Zuo et al., 2010; Scholkmann et al., 2014; Chen and Glover, 2015). The Mayer wave $(\sim 0.1 \mathrm{~Hz})$ was removed by using a $0.1 \mathrm{~Hz}$ notch filter
(Supplementary Figure 1C; Yucel et al., 2016). In step 3, the $\mathrm{OD}$ data were converted into $\mathrm{HbO}$ and $\mathrm{HbR}$ concentrations by applying the modified Beer-Lambert law (Supplementary Figure 1D; Scholkmann et al., 2014). In step 4, to further extract the functional brain activity, the hemodynamic modality separation (HMS) algorithm was used and the neural activity related $\mathrm{HbO}$ and $\mathrm{HbR}$ were obtained (Supplementary Figure 1E; Yamada et al., 2012).

\section{HbO Power Calculation of Each Epoch}

After removing the motion artifacts and systemic noise by the pre-processing procedure, the $\mathrm{HbO}$ and $\mathrm{HbR}$ concentration with high SNR were obtained. Considering that $\mathrm{HbO}$ concentration proved to be more reliable and sensitive than $\mathrm{HbR}$ in the previous studies (Strangman et al., 2002), HbO signals were used for further analysis. Considering that the root-mean-square (RMS) amplitude of the time domain signal represented the integrated amplitude of major frequencies (Chuang et al., 2008), the RMS of $\mathrm{HbO}$ concentration was calculated as $\mathrm{HbO}$ power to quantify the cortical response intensity during acupuncture by the following steps. Firstly, the sliding window technique with the moving window length of $5 \mathrm{~s}$ without overlap was used to divide the $\mathrm{HbO}$ into epochs (Baczkowski et al., 2017). Secondly, to remove the outlier epoch and improve the SNR, the noisy outlier epoch was rejected if its absolute $\mathrm{Z}$-score of $\mathrm{HbO}$ concentration was larger than 3. Finally, the $\mathrm{HbO}$ power of each epoch was calculated by using the RMS method (Supplementary Figure 1F).

\section{Hemodynamic Responses Analysis}

To compare the differences between different conditions, averaged mean time series of $\mathrm{HbO}$ concentration and $\mathrm{HbO}$ power were computed across all subjects, which was also visualized by a typical channel (Figures 4A,B,D). To further assess if there existed significant difference for different conditions, the one-way analysis of variance (ANOVA) was conducted on the $\mathrm{HbO}$ power data with different conditions (pre-acupuncture rest control, premanipulation tactile control, acupuncture manipulation, postmanipulation tactile control, and post-acupuncture rest control) as a factor (Figure 4C), in which the data samples were each condition's $\mathrm{HbO}$ power data of all epochs across all subjects. The post-hoc $t$-tests were conducted when the main effects or interactions in the ANOVA were significant. To minimize the risk of type I errors, the $p$-values were corrected with FDR correction for multiple comparisons. To verify the validity of the statistical results, the statistical power was also calculated by using the free software $G^{*}$ power. The above statistical analysis was computed using MATLAB ${ }^{\mathrm{TM}}$ 17.0 (MathWorks, United States) software.

\section{Statistical Mapping for Acupuncture Responsive Area}

To investigate the significant acupuncture responsive areas at the group level, the $\mathrm{HbO}$ power data of all epochs within different conditions across all subjects were taken as samples for statistical analysis. Considering that noisy outlier epochs were rejected previously and that the duration of different conditions varied, the data sample size of different conditions was unbalanced. 


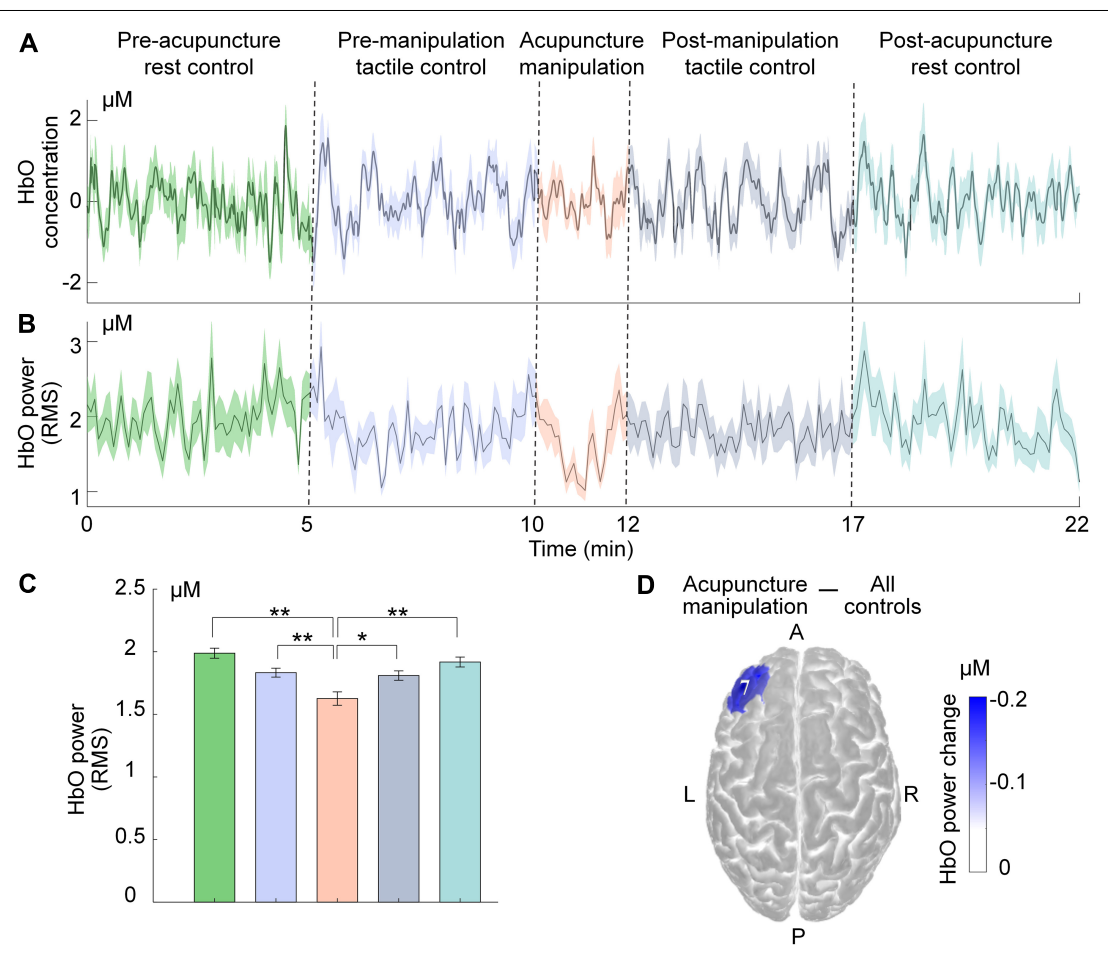

FIGURE 4 | Decrease of $\mathrm{HbO}$ power during acupuncture manipulation on a typical channel of PFC. (A,B) Time series of group-level subjects' averaged $\mathrm{HbO}$ concentration and $\mathrm{HbO}$ power under different conditions (mean $\pm \mathrm{SEM}$; subjects, $n=17$, solid line for group-level averaged value, filled color for SEM). (C) Statistical comparisons of group-level subjects' averaged HbO power for different conditions (mean $\pm \mathrm{SEM}$, one-way ANOVA, post-hoc $t$-tests with FDR correction for multiple comparisons, ${ }^{\star \star} p<0.01$ and statistical power $>0.95,{ }^{\star} p<0.05$ and statistical power $>0.8$ ). (D) Visualization of the typical channel's $\mathrm{HbO}$ power change between acupuncture manipulation and all controls (averaging four control sets data).

Therefore, two-sample $t$-tests were conducted on the HbO power data between acupuncture manipulation and all controls for each channel. To minimize the risk of type I errors, the $p$-values were corrected with FDR correction for multiple comparisons and were visualized on the standard brain template (Figure 5A). The set of significant acupuncture responsive channels $(p<0.05)$ were defined as acupuncture responsive areas (Supplementary Figure 2A) that could be divided into the PFC area and motor area according to the label of each channel. As a comparison, twosample $t$-tests were also conducted between tactile controls and rest controls (Figure 5B). In addition, to map the hemodynamic responses strength during acupuncture, the difference of the group averaged $\mathrm{HbO}$ power between acupuncture manipulation and all controls was calculated as the $\mathrm{HbO}$ power change. To verify the validity of the statistical results, the statistical power was also calculated by using the free software $\mathrm{G}^{*}$ power. Then, HbO power change was visualized for each channel on the standard brain template (Supplementary Figure 2B).

\section{Correlation Analysis Between Hemodynamic Responses and Behavioral Performances}

To reveal the relationship between hemodynamic responses and acupuncture behavior performances for the acupuncture responsive areas, Pearson's correlation and linear regression were performed between the deqi behavior data and $\mathrm{HbO}$ power change for all acupuncture significant responsive channels from the PFC and motor areas, respectively. To verify the validity of

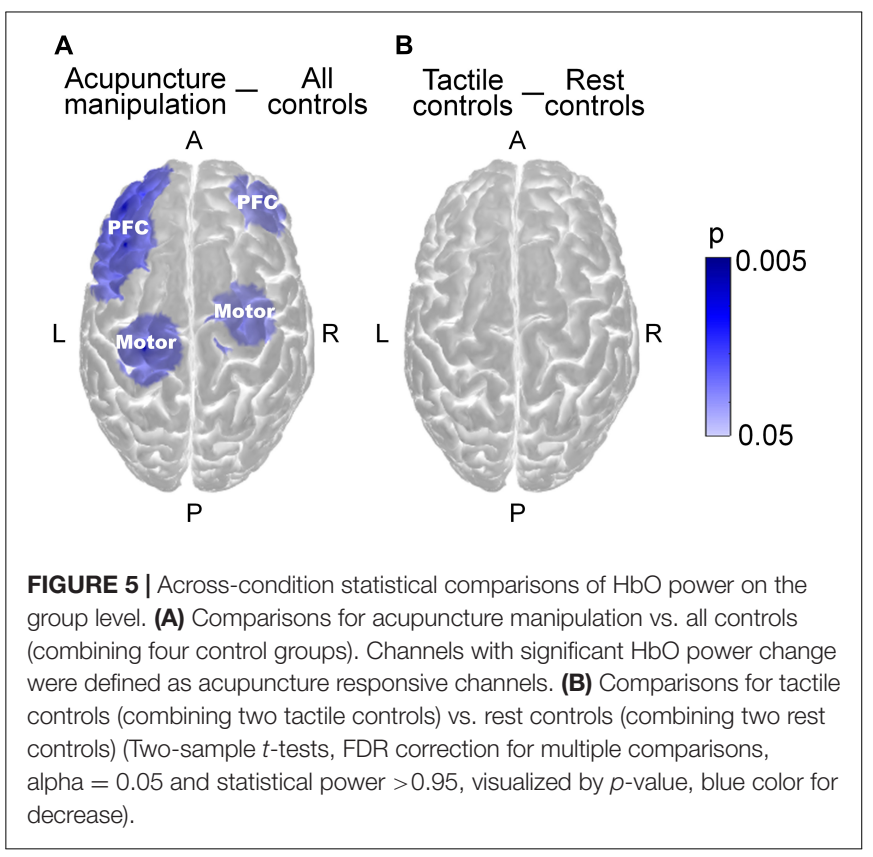



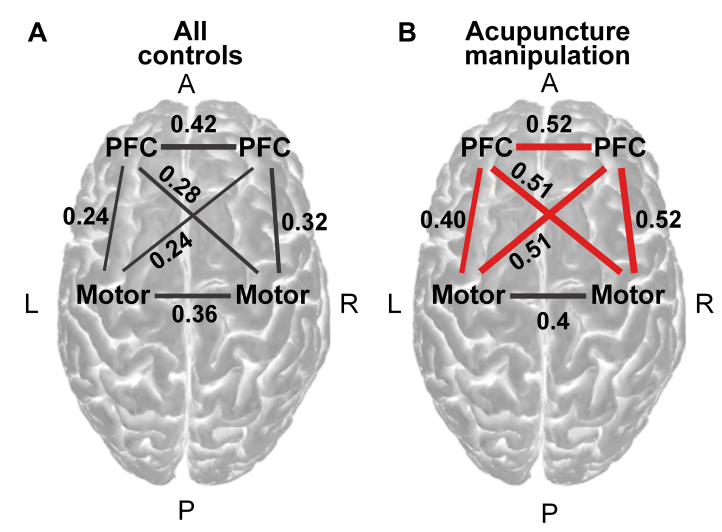

FIGURE 6 | Functional connectivity analysis across all acupuncture with deqi responsive channels on the group level. (A) Region-to-region functional connectivity under all controls (averaging four controls, permutation test, $p<0.001)$. (B) Region-to-region functional connectivity under acupuncture manipulation (permutation test, $p<0.001$ ). Red line indicates the significantly increased connections of acupuncture manipulation compared with all controls (paired $t$-tests, FDR corrected, alpha $=0.05$ and statistical power $>0.95)$. The magnitude of the connectivity value was indicated by the line width. PFC $=$ prefrontal cortex.

the correlation results, the statistical power was also calculated by using the free software $\mathrm{G}^{*}$ power. The previously rejected noisy channels were not included during the correlation analysis. For the linear regression, the 95\% confidence intervals for the mean of the polynomial evaluation were computed and visualized (Supplementary Figure 3).

\section{Functional Connectivity and Statistical Analysis}

To investigate the information flows in significant acupuncture responsive areas, functional connectivity analysis was conducted using the following steps.

Step 1, to obtain the subject level channel-to-channel functional connectivity, the significant acupuncture responsive channels were paired off to calculate the Pearson's correlation coefficients for each subject (Zhang et al., 2011). The whole time series' $\mathrm{HbO}$ concentration of acupuncture manipulation and all controls were used in the functional connectivity analysis, respectively. The previously rejected noisy channels were not included during this analysis.

Step 2, to further determine the significant channel-to-channel functional connectivity for each subject, a non-parametric permutation test was used as follows (van den Heuvel et al., 2019). Each channel's $\mathrm{HbO}$ concentration time series data were divided into $2 \mathrm{~s}$ epochs for each subject's acupuncture manipulation and all controls. Then, a 1,000-times permutation resampling method (the channel pairs' corresponding epochs were shuffled randomly) to determine the significant functional connectivity $(p<0.001)$.

Step 3, each subject's significant functional connectivity data were then subjected to Fisher's r-to-z transformation to yield variants from the normal distribution (Ghafoor et al., 2019).
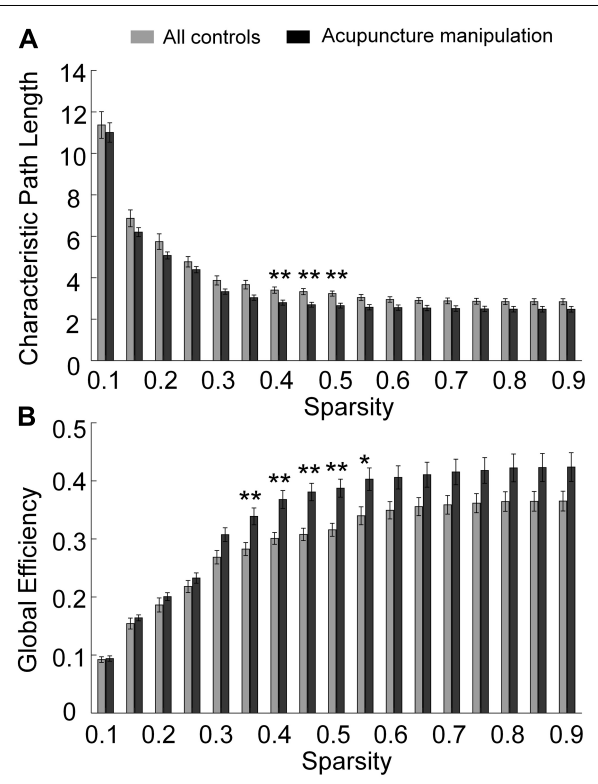

FIGURE 7 | Statistical comparations of global network metrics for acupuncture manipulation vs. all controls with different sparsity thresholds. (A) Shortest path length. (B) Global efficiency. (paired $t$-tests, ${ }^{* \star} p<0.01$ and statistical power $>0.95,{ }^{\star} p<0.05$ and statistical power $>0.8$, subjects, $n=17$, sparsity range $=[0.1,0.9]$, sparsity step-size $=0.05)$.

To obtain the group level's channel-to-channel functional connectivity matrices, then $z$-values were averaged across all subjects, and the group-level $z$-values were converted back into $r$-values via Fisher's z-to-r inverse transformation, which was in line with previous studies (Zhu et al., 2017; Cai et al., 2018; Supplementary Figures 4A,B).

Step 4, to obtain the group level region-to-region functional connectivity, subject level region-to-region functional connectivity data were firstly computed by averaging $r$-values of pair-wise channels within the same region by the previous Fisher's r-to-z and z-to-r transformation method. Then, the group level region-to-region functional connectivity data were calculated by averaging the region-to-region functional connectivity data of all subjects, which were finally visualized on the standard brain template (Figures 6A,B and Supplementary Figures 4C,D).

Step 5, to reveal the significant group level region-to-region functional connectivity between acupuncture manipulation and all controls, paired $t$-tests were conducted on subject level channel-to-channel functional connectivity data within the same region across all subjects. To verify the validity of the statistical results, the statistical power was also calculated by using the free software $G^{*}$ power. Significant increased region-to-region functional connectivity was visualized as a red line (Figure 6B).

Step 6, to further compare the functional connectivity changes of different region pairs between acupuncture manipulation and all controls (Supplementary Figure 5), paired $t$-tests were conducted on subject level channel-to-channel functional connectivity data within different region pairs (PFC-Motor, PFCPFC, and Motor-Motor) across all subjects. 


\section{Network Topological Property Analysis}

After calculating each subject's correlation coefficients for the pair-wise channels from the acupuncture responsive areas, the correlation coefficients matrix was obtained for each subject. Based on the calculated correlation coefficients matrix, the functional brain network could be reconstructed between acupuncture manipulation and all controls for each subject, in which channels served as nodes and functional connectivity between channels served as edges. The previously rejected noisy channels were not included during this analysis.

To further reveal the acupuncture's modulation effect on the functional brain network, the graph theory was utilized to describe the functional network's topological structure changes between different conditions (Yu et al., 2018, 2019). The shortest path length and global efficiency were calculated and used as network metrics for each subject, in which the correlation coefficients matrix was thresholded over a range of sparsity (from 0.1 to 0.9 with step-size of 0.05 ) to investigate the relationship between network metrics and sparsity (Figure 7; Niu et al., 2013; Geng et al., 2017). In addition, other network metrics, including the local efficiency, clustering coefficient, and small-world, were also calculated, presented in Supplementary Figure 6. These network metrics were computed by a freely available MATLAB ${ }^{\text {TM }}$ toolbox, GRaph thEoreTical Network Analysis (GRETNA) (Wang et al., 2015).

For the network with $\mathrm{N}$ nodes and $\mathrm{K}$ edges, the shortest path length of the network (Figure 7A) was defined as the average of the shortest path lengths between all pairs of nodes in the network (Latora and Marchiori, 2003):

$$
L_{p}=\frac{1}{N(N-1)} \sum_{i \neq j \in G} d_{i j},
$$

where $d_{i j}$ was the shortest path length between node $i$ and node $j$, which meant the minimum number of edges included in the path that connected these two nodes.

The global efficiency (Figure 7B) was defined as (Latora and Marchiori, 2001):

$$
E_{g l o b}=\frac{1}{N(N-1)} \sum_{i \neq j \in G} \frac{1}{d_{i j}},
$$

where $d_{i j}$ is the shortest path length between node $i$ and node $j$.

To reveal the significant difference between acupuncture manipulation and all controls, paired $t$-tests were performed on all subjects' global network metric data for different conditions (Figure 7). To verify the validity of the statistical results, the statistical power was also calculated by using the free software $\mathrm{G}^{*}$ power.

\section{RESULTS}

\section{Behavior deqi Performances Under Different Conditions}

To verify the behavioral effectiveness of acupuncture manipulation, paired $t$-tests with FDR correction were used to compare the deqi index for different conditions (Figure 3 and Supplementary Table 1). The subjects' deqi index of acupuncture manipulation was significantly larger than that of pre-manipulation tactile control and post-manipulation tactile control ${ }^{* *} p<0.01$ and statistical power $>0.95$ ) (Figure 3). In comparison, there was no significant difference between pre-manipulation tactile control and post-manipulation tactile control (n.s.) (Figure 3). These results indicated that acupuncture manipulation could cause significant deqi behavioral effects compared to tactile controls.

\section{Decreased HbO Responses by Acupuncture Manipulation}

To reveal the deqi modulation effects of acupuncture on the cerebral cortex, the $\mathrm{HbO}$ concentration was firstly calculated after pre-processing of fNIRS data. Subjectaveraged group-level results showed reduced amplitude of fluctuation for $\mathrm{HbO}$ concentration during acupuncture manipulation, which was shown by a typical channel from PFC (Figure 4A). Furthermore, the RMS of $\mathrm{HbO}$ concentration was computed as the $\mathrm{HbO}$ power index to quantify the $\mathrm{HbO}$ fluctuation energy. The typical channel's subject-averaged results also showed reduced $\mathrm{HbO}$ power during acupuncture manipulation (Figure 4B). Furthermore, one-way ANOVA on the $\mathrm{HbO}$ power shown a significant difference between the five conditions (Figure 4C). The post-hoc $t$-tests with FDR correction shown that acupuncture manipulation's $\mathrm{HbO}$ power was significantly smaller than the other four controls [pre-acupuncture rest control, ${ }^{* *} p<0.01$ and statistical power $>0.95$; pre-manipulation tactile control, ${ }^{* *} p<0.01$ and statistical power $>0.95$; post-manipulation tactile control, $p<0.05$ and statistical power $>0.8$; postacupuncture rest control, ${ }^{* *} p<0.01$ and statistical power $>0.95$ ] (Figure 4C). This typical channel's $\mathrm{HbO}$ power change between acupuncture manipulation and all controls was computed and visualized on the standard brain template (Figure 4D).

\section{Acupuncture Responsive Area}

To reveal the acupuncture with deqi responsive areas, the $\mathrm{HbO}$ power response patterns were examined across all channels from seventeen subjects (Figure 5A and Supplementary Figure 2). Channels from the bilateral PFC and motor cortex showed the strongest decreased $\mathrm{HbO}$ power change (Supplementary Figure 2B). Among them, the acupuncture responsive areas were identified as those channels that had significantly lower $\mathrm{HbO}$ power between acupuncture manipulation and all controls (acupuncture responsive channels, $n=8 ; p<0.05$; twosample $t$-tests with FDR correction). The acupuncture with deqi responsive channels were distributed over the PFC and the motor cortex bilaterally, which was shown on the standard brain template (Figure 5A) and was indicated with channel labels (Supplementary Figure 2A). For the two different controls, there was no significantly changed $\mathrm{HbO}$ power channel by comparing the $\mathrm{HbO}$ power change between tactile controls and rest controls (Figure 5B). 


\section{The PFC's Hemodynamic Response Had a Positive Correlation Trend With Behavior Score}

To investigate whether there is a relationship between hemodynamic response and behavior performance for acupuncture with deqi responsive areas, Pearson's correlation was performed across all subjects for the bilateral PFC and the bilateral motor cortex, respectively. Interestingly, the $\mathrm{HbO}$ power change (acupuncture manipulation - all controls) of the acupuncture with deqi responsive channels from the PFC had a positive correlation trend with acupuncture behavior deqi index $(r=0.234, p<0.05$ and statistical power $>0.50)$ (Supplementary Figure 3A). In contrast, there was no significant correlation for the acupuncture responsive channels from the motor cortex $(r=-0.073$, n.s.) (Supplementary Figure 3B). These results showed that the PFC's HbO responses could predict the subject's behavior performance during acupuncture manipulation.

\section{The PFC Related Connection Showed Significantly Increased Functional Connectivity}

To reveal the information flows among key nodes in the network, functional connectivity analysis was used to explore the neural interactions between the acupuncture with deqi responsive channel pairs. The significant functional connectivity edges (permutation test, $p<0.001$ ) were calculated and visualized for all controls (Figure 6A and Supplementary Figures 4A,C) and acupuncture manipulation (Figure 6B and Supplementary Figures 4B,D). It was shown that there was overall increased functional connectivity strength for acupuncture manipulation, compared with all controls (Figures 6A,B). Importantly, channels over the PFC were found to have significantly enhanced connections with the motor and the PFC under acupuncture manipulation compared with all controls (paired t-tests, alpha $=0.05$ and statistical power $>0.95$ ) (Figure 6B), which was also verified by subsequent results.

Compared with all controls, PFC-Motor and PFC-PFC showed significantly higher functional connectivity strength under acupuncture manipulation (paired $t$-tests, ${ }^{* *} p<0.01$ and statistical power $>0.95$ ) (Supplementary Figures 5A,B). In contrast, the Motor-Motor connection showed no significant difference between acupuncture manipulation and all controls (paired $t$-tests, n.s.) (Supplementary Figure 5C).

\section{Topological Properties of the Cortical Functional Network}

To further reveal acupuncture with deqi's modulation effects on the functional brain network, graph theory analysis was performed on the functional connectivity network with the acupuncture with deqi responsive channels as nodes. The topological properties of the network were revealed by different global network metrics with different sparsity thresholds (Figure 7). To reveal the information transmission ability of the network, the shortest path length and global efficiency were showed. Compared with all controls, the shortest path length parameter showed a lower tendency. It was significantly lower within $0.4-0.5$ sparsity range for acupuncture manipulation compared with all controls (paired $t$-tests, alpha $=0.05$ and statistical power $>0.95)$ (Figure 7A). On the contrary, the global efficiency parameter showed a higher pattern and was significantly higher within the 0.35-0.55 sparsity range for acupuncture manipulation (paired $t$-tests, alpha $=0.05$ and statistical power $>0.95$ ) (Figure 7B). In addition, other network metrics, including the local efficiency, clustering coefficient, and small-world, did not have a significant difference between acupuncture manipulation and all controls (Supplementary Figure 6).

\section{DISCUSSION}

\section{A Cooperative PFC-Motor Functional Network for Acupuncture}

As the basic acupuncture unit for regulating the central nervous system (Zhang et al., 2012), the Hegu acupoint was employed in this study to explore the neuromodulation effects of acupuncture. Different from most previous findings of studies examining the involvement of subcortical localized brain areas during acupuncture (Chen et al., 2006; Dhond et al., 2008; Yu et al., 2017), our results revealed a distributed and cooperative cortical acupuncture with deqi network with the PFC and the motor cortex as nodes.

Firstly, acupuncture manipulation caused significant behavioral deqi effects compared to tactile controls (Figure 3). Secondly, by investigating the effects of acupuncture at the Hegu acupoint with deqi on cerebral hemodynamic responses, we found the decreased $\mathrm{HbO}$ responses could be a potential biomarker for acupuncture (Figure 4 and Supplementary Figure 2). Thirdly, the bilateral PFC and the motor cortex were found to be acupuncture responsive nodes (Figure 5), and the PFC's hemodynamic responses were significantly correlated with the behavior deqi index (Supplementary Figure 3A). Interestingly, the PFC-related connections in the acupuncture network showed significantly increased functional connectivity during acupuncture manipulation (Figure 6 and Supplementary Figure 5). Finally, the topological properties of the acupuncture network showed that the network efficiency was improved by acupuncture with deqi (Figure 7). Taken together, these results indicate that there is a cooperative PFC-Motor acupuncture functional network, which could provide more neuroimaging evidence for explaining acupuncture with deqi's neuromodulation effects on the human brain.

\section{Decreased HbO Response by Acupuncture Manipulation}

By analyzing the hemodynamic responses recorded by fNIRS (Figure 4 and Supplementary Figure 1), the bilateral PFC and the motor cortex were found to show decreased $\mathrm{HbO}$ responses during acupuncture manipulation (Figure $\mathbf{5}$ and Supplementary Figure 2). Previous fMRI-fNIRS simultaneous recording studies have proved that the $\mathrm{HbO}$ signal is positively 
correlated with the blood-oxygen-level-dependent (BOLD) fMRI signal (Strangman et al., 2002). In addition, the deactivation of the BOLD signal recorded by fMRI was further proved to reflect the suppression of neuronal activity by previous studies (Shmuel et al., 2002; Stefanovic et al., 2004). These findings suggest that the observed decrease of $\mathrm{HbO}$ response from the bilateral PFC and motor cortex could reflect the suppression of underlying neural activities. Moreover, the bilateral PFC and motor cortex could be network nodes during acupuncture manipulation.

\section{Acupuncture Modulates the PFC and Regulates the Cognition Function}

In this study, the bilateral PFC showed significantly decreased $\mathrm{HbO}$ responses during acupuncture manipulation (Figure 5 and Supplementary Figure 2), which indicates that acupuncture could modulate the hemodynamic responses of bilateral PFC. The PFC is an important region for high-level cognition processing, which can provide top-down regulation of attention, emotion, and cognitive control (Arnsten and Rubia, 2012). It has been postulated that acupuncture manipulation could regulate cognitive functions by modulating the bilateral PFC responses, as evidenced by previous studies. Firstly, previous studies have shown that the high-level cognitive functions could be modulated by using neuromodulation methods such as TMS (Curtin et al., 2019a), tDCS (Di Rosa et al., 2019), and acupuncture stimulation (Zheng et al., 2018). For example, the previous fNIRS-TMS and fNIRS-tDCS studies found that neuromodulation could improve cognitive performances during working memory tasks (Di Rosa et al., 2019) and speed of processing (SOP) cognitive tasks (Curtin et al., 2019a), which were both evidenced by the reduced amplitude of $\mathrm{HbO}$ concentration in the PFC (Curtin et al., 2019a; Di Rosa et al., 2019). Secondly, the dorsolateral prefrontal cortex (DLPFC) is a key node of the central executive network (CEN), which is responsible for high-level cognitive and attentional control (Menon and Uddin, 2010). Therefore, it could be further speculated that the CEN could be modulated by acupuncture manipulation, which helps to improve the brain's cognitive functions. Finally, previous fMRI studies found that acupuncture modulated BOLD responses and could improve cognition performances for stroke patients (Zhang et al., 2014), mild cognitive impairment patients (Feng et al., 2012), and Alzheimer's disease (AD) patients (Wang et al., 2014; Zheng et al., 2018), which supported our fNIRS findings. In addition, although acupuncture responsive channels were distributed over the PFC and the motor cortex bilaterally, the PFC showed a left hemisphere dominant response tendency. As all subjects were right-hand controlled in this study, we speculate that it might be related to right-handedness, which needs to be explored in future studies. By modulating bilateral PFC responses, acupuncture manipulation could help regulate cognitive functions.

\section{The PFC's HbO Response as an Objective Biomarker for Acupuncture}

The needling deqi sensation that subjects experienced is considered a valuable behavioral index in evaluating the effect of acupuncture in TCM (Yang et al., 2013). However, the current behavioral evaluation of deqi is subjective and insufficient and lacks an objective neural biomarker. In this fNIRS study, we found that the PFC showed a positive correlation trend between the acupuncture deqi behavior index and the $\mathrm{HbO}$ power change (acupuncture manipulation - all controls) (Supplementary Figure 3), which meant that the PFC's HbO responses could predict the subject's behavior performance during acupuncture manipulation. This result showed that the PFC's HbO response could potentially be used as an objective neural biomarker to evaluate the effects of acupuncture at the Hegu acupoint.

\section{Modulation of the Motor Cortex and the Analgesic Effect of Acupuncture}

In this study, the bilateral motor cortex showed significantly decreased $\mathrm{HbO}$ responses during acupuncture manipulation (Figure 5 and Supplementary Figure 2), which indicated that acupuncture could modulate the hemodynamic responses of the bilateral motor cortex. Considering that the motor cortex is an important region for pain processing and control (Volz et al., 2015; Lopes et al., 2019) we argue that by modulating the bilateral motor cortex's $\mathrm{HbO}$ responses, acupuncture manipulation could produce analgesic effects. Firstly, it has been well known that the motor cortex has the role of pain control (Ostergard et al., 2014), and electrical stimulation in the motor cortex could treat intractable pain syndromes effectively (Arle and Shils, 2008; Henssen et al., 2019). Secondly, previous fNIRS studies also proved that analgesic effects could be delivered during acupuncture manipulation, in which the decreased hemodynamic responses of the motor cortex were observed (Takamoto et al., 2010; Rojas et al., 2019). The decreased motor activities showed by the above fNIRS studies were in line with our findings. Taking together, acupuncture manipulation could suppress the excessive motor cortex activity, which helps explain the analgesic effects of acupuncture.

\section{The Bilateral PFC as Network Hub During Acupuncture}

Here, the bilateral PFC was found to have significantly increased functional connections with the motor cortex (PFC-Motor) and with the PFC cortex (PFC-PFC) during acupuncture manipulation compared with all controls (Figure 6 and Supplementary Figure 5). We argue that the bilateral PFC works as a network hub in the cooperative PFC-Motor functional network during acupuncture manipulation. We further postulate that the increased PFC-related functional connectivity could not only improve the human cognitive ability but also produce an analgesic effect through acupuncture manipulation.

Functional connectivity has been widely used to reveal cognition functions (Zeng et al., 2012; Cohen, 2018). Moreover, previous studies showed that the human cognitive ability could be improved during acupuncture with deqi, which was evidenced by the increased functional connectivity (Dhond et al., 2008; Bai et al., 2009; Yu et al., 2017). In this study, the observed increased functional connectivity with PFC as node indicated that the longdistance information transmissions with PFC network hub were enhanced. Therefore, acupuncture with deqi could strengthen the 
communications between remote brain areas within the largescale network and improve cognitive function.

A previous study showed that the PFC-motor's functional connection had the role of pain control (Peng et al., 2019). In our study, acupuncture manipulation significantly improved the PFC-Motor connections. Therefore, we postulate that the motor cortex might receive increased top-down modulation from the PFC, which could produce the analgesic effect through the recruitment of descending pain inhibition system (Peng et al., 2019). The bilateral PFC is a network hub in the cooperative PFC-Motor functional network for acupuncture. The increased functional connections of bilateral PFC by acupuncture with deqi might play a role in improving human cognitive ability and produces analgesic effects.

\section{Acupuncture Improves the Global Efficiency of the PFC-Motor Functional Network}

Compared with all controls, the topological properties of the network showed a lower shortest path length (Figure 7A) and higher global efficiency (Figure 7B) during acupuncture manipulation (Figure 7). We argue that acupuncture manipulation could improve the information transmission of the PFC-Motor functional network. Firstly, global efficiency is a global parameter to describe the network's capacity for parallel information exchanging between nodes via multiple edges (Rubinov and Sporns, 2010). A higher global efficiency shows that PFC and Motor cortex are well integrated and that information transfer over the PFC-Motor network is more efficient by acupuncture manipulation (Fleischer et al., 2019). Secondly, the path length is a measure of processing steps along the path of information transfer between different nodes (Rubinov and Sporns, 2010). Since fewer numbers of processing steps denote a more rapid and accurate information communication, a lower shortest path length indicates a higher level of communication efficiency among the PFC-Motor network by acupuncture (Kaiser and Hilgetag, 2006).

We further postulate that acupuncture manipulation could improve cognitive ability by improving the global efficiency of the network. Firstly, the functional brain network attained a smaller path length and a larger global efficiency under acupuncture manipulation, indicating that the enhancement of the processing efficiency in the brain network (Yu et al., 2017). Secondly, a previous study found that acupuncture could improve the overall processing efficiency of the brain network among MCI patients, reflecting the improvement of cognitive function by acupuncture manipulation (Ghafoor et al., 2019). Acupuncture manipulation improves the information transmission of the PFC-Motor functional network and could enhance the human cognitive ability, which could also explain the increased functional connections of the PFC-Motor network (Figure 6 and Supplementary Figure 5).

\section{Limitations and Future Works}

There exist several potential limitations to this study. Firstly, more participants should be recruited in future studies to reach a higher statistical power, and provide more evidence for further exploring the relationship between hemodynamic response and acupuncture behavior performance. For example, although the bilateral PFC's hemodynamic response showed a positive correlation trend with acupuncture behavioral performance (Supplementary Figure 3), the statistical power should be further validated by recruiting more participants in the following study. Secondly, although a quasi-experimental design and pre/posttactical/rest controls were employed in this study, a more rigorous parallel control experiment design should be conducted in the future, which could make the conclusion more convincing. Thirdly, this study explored the effects of deqi on cortical networks, future studies should be conducted with patients to add more neuroimaging evidence for explaining the clinical therapeutic effects of acupuncture with deqi. Fourthly, due to the limited channel number of the current fNIRS device, the channel layout of fNIRS could not have complete coverage of the whole brain. In the future, a whole-brain coverage fNIRS device should be used to explore the neural mechanism of acupuncture further. Finally, our study used Pearson's correlation coefficients as functional connectivity, which could not reveal the causal relationship between brain regions. In future studies, Granger causality analysis could be used to further quantify the strength of directed effective connectivity between brain regions.

\section{CONCLUSION}

Despite acupuncture's long history and public acceptance, the underlying neural mechanism of acupuncture's neuromodulation effects on the human brain remain largely unclear. By using multi-channel fNIRS recordings on the human brain, our study found that acupuncture modulated a distributed and cooperative PFC-Motor cortical network with the bilateral PFC and the motor cortex as key nodes. Taking the basic neural acupuncture unit Hegu acupoint as an example, acupuncture manipulation not only modulated the hemodynamic responses of the bilateral PFC and motor cortex but also regulated the functional connectivity and efficiency of the PFC-Motor cortical network. Our study contributes objective neuroimaging evidence for explaining acupuncture's neuromodulation effects on the human brain, which also contributes to Traditional Chinese Medicine.

\section{DATA AVAILABILITY STATEMENT}

The data and codes that support the findings of this study are available upon request.

\section{ETHICS STATEMENT}

The studies involving human participants were reviewed and approved by the Institutional Review Board and Ethics Committee of Tianjin University. The patients/participants provided their written informed consent to participate in this study. 


\section{AUTHOR CONTRIBUTIONS}

XS designed and conceptualized the research. KZ, LZ, and XS collected the data. SX, SL, LZ, and XS analyzed the data. SX and XS wrote the manuscript. KZ and SL helped with manuscript revision. XS and DM supervision. All authors contributed to the article and approved the submitted version.

\section{FUNDING}

This work was supported by the National Natural Science Foundation of China (No. 61906132 to XS and No. 81925020 to DM), China Postdoctoral Science Foundation (No. 2020T130092ZX to KZ), Key Project \& Team Program of

\section{REFERENCES}

Aasted, C. M., Yucel, M. A., Cooper, R. J., Dubb, J., Tsuzuki, D., Becerra, L., et al. (2015). Anatomical guidance for functional near-infrared spectroscopy: AtlasViewer tutorial. Neurophotonics 2:020801. doi: 10.1117/1.NPh.2.2.020801

Arle, J. E., and Shils, J. L. (2008). Motor cortex stimulation for pain and movement disorders. Neurotherapeutics 5, 37-49. doi: 10.1016/j.nurt.2007.11.004

Arnsten, A. F. T., and Rubia, K. (2012). Neurobiological circuits regulating attention, cognitive control, motivation, and emotion: disruptions in neurodevelopmental psychiatric disorders. J. Am. Acad. Child Adolesc. Psychiatry 51, 356-367. doi: 10.1016/j.jaac.2012.01.008

Asghar, A. U., Green, G., Lythgoe, M. F., Lewith, G., and MacPherson, H. (2010). Acupuncture needling sensation: The neural correlates of deqi using fMRI. Brain Res. 1315, 111-118. doi: 10.1016/j.brainres.2009.12.019

Baczkowski, B. M., Johnstone, T., Walter, H., Erk, S., and Veer, I. M. (2017). Slidingwindow analysis tracks fluctuations in amygdala functional connectivity associated with physiological arousal and vigilance during fear conditioning. Neuroimage 153, 168-178. doi: 10.1016/j.neuroimage.2017.03.022

Bai, L. J., Qin, W., Tian, J., Dai, J. P., and Yang, W. H. (2009). Detection of dynamic brain networks modulated by acupuncture using a graph theory model. Prog. Nat. Sci. Mater. 19, 827-835. doi: 10.1016/j.pnsc.2008.09.009

Bärnighausen, T., Tugwell, P., Røttingen, J. A., Shemilt, I., Rockers, P., Geldsetzer, P., et al. (2017). Quasi-Experimental study designs series-paper 4: uses and value. J. Clin. Epidemiol. 89, 21-29.

Bassett, D. S., and Sporns, O. (2017). Network neuroscience. Nat. Neurosci. 20, 353-364. doi: 10.1038/nn.4502

Behrendt, H. F., Firk, C., Nelson, C. A., and Perdue, K. L. (2018). Motion correction for infant functional near-infrared spectroscopy with an application to live interaction data. Neurophotonics 5:015004. doi: 10.1117/1.NPh.5.1. 015004

Boas, D. A., Culver, J. P., Stott, J. J., and Dunn, A. K. (2002). Three dimensional Monte Carlo code for photon migration through complex heterogeneous media including the adult human head. Opt. Express 10, 159-170. doi: 10.1364/Oe.10. 000159

Cai, L., Dong, Q., and Niu, H. J. (2018). The development of functional network organization in early childhood and early adolescence: A resting-state fNIRS study. Dev. Cogn. Neurosci. 30, 223-235. doi: 10.1016/j.den.2018.03.003

Chen, A. C. N., Liu, F. J., Wang, L., and Arendt-Nielsen, L. (2006). Mode and site of acupuncture modulation in the human brain: 3D (124-ch) EEG power spectrum mapping and source imaging. Neuroimage 29, 1080-1091. doi: 10. 1016/j.neuroimage.2005.08.066

Chen, J. Y. E., and Glover, G. H. (2015). BOLD fractional contribution to restingstate functional connectivity above $0.1 \mathrm{~Hz}$. Neuroimage 107, 207-218. doi: 10 . 1016/j.neuroimage.2014.12.012

Chuang, K. H., van Gelderen, P., Merkle, H., Bodurka, J., Ikonomidou, V. N., Koretsky, A. P., et al. (2008). Mapping resting-state functional connectivity using perfusion MRI. Neuroimage 40, 1595-1605. doi: 10.1016/j.neuroimage. 2008.01.006
Tianjin City (No. XC202020 to XS), and Tianjin University (No. 2020XRY-0015 to XS).

\section{ACKNOWLEDGMENTS}

The authors would like to thank all the participants.

\section{SUPPLEMENTARY MATERIAL}

The Supplementary Material for this article can be found online at: https://www.frontiersin.org/articles/10.3389/fnins. 2021.693623/full\#supplementary-material

Cohen, J. R. (2018). The behavioral and cognitive relevance of time-varying, dynamic changes in functional connectivity. Neuroimage 180, 515-525. doi: 10.1016/j.neuroimage.2017.09.036

Curtin, A., Ayaz, H., Tang, Y. Y., Sun, J. F., Wang, J. J., and Tong, S. B. (2019a). Enhancing neural efficiency of cognitive processing speed via training and neurostimulation: An fNIRS and TMS study. Neuroimage 198, 73-82. doi: 10.1016/j.neuroimage.2019.05.020

Curtin, A., Tong, S. B., Sun, J. F., Wang, J. J., Onaral, B., and Ayaz, H. (2019b). A Systematic Review of Integrated Functional Near-Infrared Spectroscopy (fNIRS) and Transcranial Magnetic Stimulation (TMS) Studies. Front. Neurosci. 13:84. doi: 10.3389 /fnins.2019.00084

Dhond, R. P., Yeh, C., Park, K., Kettner, N., and Napadow, V. (2008). Acupuncture modulates resting state connectivity in default and sensorimotor brain networks. Pain 136, 407-418. doi: 10.1016/j.pain.2008.01.011

Di Lorenzo, R., Pirazzoli, L., Blasi, A., Bulgarelli, C., Hakuno, Y., Minagawa, Y., et al. (2019). Recommendations for motion correction of infant fNIRS data applicable to multiple data sets and acquisition systems. Neuroimage 200, 511-527. doi: 10.1016/j.neuroimage.2019.06.056

Di Rosa, E., Brigadoi, S., Cutini, S., Tarantino, V., Dell'Acqua, R., Mapelli, D., et al. (2019). Reward motivation and neurostimulation interact to improve working memory performance in healthy older adults: A simultaneous tDCS-fNIRS study. Neuroimage 202:116062. doi: 10.1016/j.neuroimage.2019.116062

Fang, J. L., Jin, Z., Wang, Y., Li, K., Kong, J., Nixon, E. E., et al. (2009). The salient characteristics of the central effects of acupuncture needling: limbicparalimbic-neocortical network modulation. Hum. Brain Mapp. 30, 1196-1206. doi: $10.1002 / \mathrm{hbm} .20583$

Feng, Y. Y., Bai, L. J., Ren, Y. S., Chen, S. J., Wang, H., Zhang, W. S., et al. (2012). FMRI connectivity analysis of acupuncture effects on the whole brain network in mild cognitive impairment patients. Magn. Reson. Imaging 30, 672-682. doi: 10.1016/j.mri.2012.01.003

Fleischer, V., Radetz, A., Ciolac, D., Muthuraman, M., Gonzalez-Escamilla, G., Zipp, F., et al. (2019). Graph theoretical framework of brain networks in multiple sclerosis: a review of concepts. Neuroscience 403, 35-53. doi: 10.1016/ j.neuroscience.2017.10.033

Geng, S. J., Liu, X. Y., Biswal, B. B., and Niu, H. J. (2017). Effect of resting-state fNIRS scanning duration on functional brain connectivity and graph theory metrics of brain network. Front. Neurosci. Switz. 11:392. doi: 10.3389/fnins. 2017.00392

Ghafoor, U., Lee, J. H., Hong, K. S., Park, S. S., Kim, J., and Yoo, H. R. (2019). Effects of acupuncture therapy on MCI patients using functional near-infrared spectroscopy. Front. Aging Neurosci. 11:237. doi: 10.3389/fnagi.2019.00237

Hakuno, Y., Hata, M., Naoi, N., Hoshino, E., and Minagawa, Y. (2020). Interactive live fNIRS reveals engagement of the temporoparietal junction in response to social contingency in infants. Neuroimage 218:116901.

Henssen, D. J. H. A., Witkam, R. L., Dao, J. C. M. L., Comes, D. J., van Walsum, A. M. V., Kozicz, T., et al. (2019). Systematic review and neural network analysis to define predictive variables in implantable motor cortex stimulation to treat chronic intractable pain. J. Pain 20, 1015-1026. doi: 10.1016/j.jpain.2019.02.004 
Hipp, J. F., Hawellek, D. J., Corbetta, M., Siegel, M., and Engel, A. K. (2012). Large-scale cortical correlation structure of spontaneous oscillatory activity. Nat. Neurosci. 15, 884-890. doi: 10.1038/nn.3101

Hui, K. K. K. S., Sporko, T. N., Vangel, M. G., Li, M., Fang, J. L., and Lao, L. X. (2011). Perception of Deqi by Chinese and American acupuncturists: a pilot survey. Chin. Med. Uk 6:2. doi: 10.1186/1749-8546-6-2

Hui, K. K. S., Liu, J., Marina, O., Napadow, V., Haselgrove, C., Kwong, K. K., et al. (2005). The integrated response of the human cerebro-cerebellar and limbic systems to acupuncture stimulation at ST 36 as evidenced by fMRI. Neuroimage 27, 479-496. doi: 10.1016/j.neuroimage.2005.04.037

Hui, K. K. S., Marina, O., Liu, J., Rosen, B. R., and Kwong, K. K. (2010). Acupuncture, the limbic system, and the anticorrelated networks of the brain. Auton. Neurosci. Basic 157, 81-90. doi: 10.1016/j.autneu.2010.03.022

Hui, K. K. S., Nixon, E. E., Vangel, M. G., Liu, J., Marina, O., Napadow, V., et al. (2007). Characterization of the "deqi" response in acupuncture. BMC Complement. Altern. Med. 7:33. doi: 10.1186/1472-6882-7-33

Huppert, T. J., Diamond, S. G., Franceschini, M. A., and Boas, D. A. (2009). HomER: a review of time-series analysis methods for near-infrared spectroscopy of the brain. Appl. Opt. 48, D280-D298. doi: 10.1364/Ao.48. $00 \mathrm{~d} 280$

Jiang, F., Yang, T., Yin, H., Guo, Y., Namba, H., Sun, Z., et al. (2018). Evidence for the use of acupuncture in treating parkinson's disease: update of information from the past 5 years, a mini review of the literature. Front. Neurol. 9:596. doi: 10.3389/fneur.2018.00596

Kaiser, M., and Hilgetag, C. C. (2006). Nonoptimal component placement, but short processing paths, due to long-distance projections in neural systems. PLoS Comput. Biol. 2:e95. doi: 10.1371/journal.pcbi.0020095

Keles, H. O., Barbour, R. L., and Omurtag, A. (2016). Hemodynamic correlates of spontaneous neural activity measured by human whole-head resting state EEG+fNIRS. Neuroimage 138, 76-87. doi: 10.1016/j.neuroimage.2016.05.058

Kong, J., Gollub, R., Huang, T., Polich, G., Napadow, V., Hui, K., et al. (2007). Acupuncture de qi, from qualitative history to quantitative measurement. J. Altern. Complem. Med. 13, 1059-1070. doi: 10.1089/acm.2007.0524

Kong, S. P., Tan, Q. W., Liu, Y., Jing, X. H., Zhu, B., Huo, Y. J., et al. (2015). Specific Correlation between the Hegu Point (LI4) and the Orofacial Part: Evidence from an fMRI Study. Evid. Based Complement. Alternat. Med. 2015:585493.

Kou, W., Gareus, I., Bell, J. D., Goebel, M. U., Spahn, G., Pacheco-Lopez, G., et al. (2007). Quantification of DeQi sensation by visual analog scales in healthy humans after immunostimulating acupuncture treatment. Am. J. Chin. Med. 35, 753-765. doi: 10.1142/S0192415x07005247

Latora, V., and Marchiori, M. (2001). Efficient behavior of small-world networks. Phys. Rev. Lett. 87:198701. doi: 10.1103/PhysRevLett.87.198701

Latora, V., and Marchiori, M. (2003). Economic small-world behavior in weighted networks. Eur. Phys. J. B 32, 249-263. doi: 10.1140/epjb/e2003-00095-5

Lee, G. E., Yun, J. M., Yang, S. B., Kang, Y., Kang, H. W., Choi, K. H., et al. (2017). Deqi induction by HT7 acupuncture alters theta and alpha band coherence in human healthy subjects. Evid. Based Complement. Alternat. Med. 2017:7107136. doi: 10.1155/2017/7107136

Legon, W., Sato, T. F., Opitz, A., Mueller, J., Barbour, A., Williams, A., et al. (2014). Transcranial focused ultrasound modulates the activity of primary somatosensory cortex in humans. Nat. Neurosci. 17, 322-329. doi: 10.1038/nn. 3620

Liang, P., Wang, Z., Qian, T., and Li, K. (2014). Acupuncture stimulation of Taichong (Liv3) and Hegu (LI4) modulates the default mode network activity in Alzheimer's disease. Am. J. Alzheimers Dis. Other Demen. 29, 739-748. doi: $10.1177 / 1533317514536600$

Lopes, P. S. S., Campos, A. C. P., Fonoff, E. T., Britto, L. R. G., and Pagano, R. L. (2019). Motor cortex and pain control: exploring the descending relay analgesic pathways and spinal nociceptive neurons in healthy conscious rats. Behav. Brain Funct. 15:5. doi: 10.1186/s12993-019-0156-0

Lund, I., and Lundeberg, T. (2016). Mechanisms of acupuncture. Acupunct. Relat. Ther. 4, 26-30. doi: 10.1016/j.arthe.2016.12.001

Menon, V., and Uddin, L. Q. (2010). Saliency, switching, attention and control: a network model of insula function. Brain Struct. Funct. 214, 655-667. doi: 10.1007/s00429-010-0262-0

Miranda, J., Lamana, S. M. S., Dias, E. V., Athie, M., Parada, C. A., and Tambeli, C. H. (2015). Effect of pain chronification and chronic pain on an endogenous pain modulation circuit in rats. Neuroscience 286, 37-44. doi: 10.1016/j. neuroscience.2014.10.049

Niu, H. J., Li, Z., Liao, X. H., Wang, J. H., Zhao, T. D., Shu, N., et al. (2013). TestRetest reliability of graph metrics in functional brain networks: a resting-state fNIRS study. PLoS One 8:e72425. doi: 10.1371/journal.pone.0072425

Okamoto, M., Tsuzuki, D., Clowney, L., Dan, H., Singh, A. K., and Dan, I. (2009). Structural atlas-based spatial registration for functional near-infrared spectroscopy enabling inter-study data integration. Clin. Neurophysiol. 120, 1320-1328. doi: 10.1016/j.clinph.2009.01.023

Ostergard, T., Munyon, C., and Miller, J. P. (2014). Motor cortex stimulation for chronic pain. Neurosurg. Clin. N. Am. 25:693. doi: 10.1016/j.nec.2014.06.004

Park, E., Kang, M. J., Lee, A., Chang, W. H., Shin, Y. I., and Kim, Y. H. (2017). Real-time measurement of cerebral blood flow during and after repetitive transcranial magnetic stimulation: A near-infrared spectroscopy study. Neurosci. Lett. 653, 78-83. doi: 10.1016/j.neulet.2017. 05.039

Patel, R., Dawidziuk, A., Darzi, A., Singh, H., and Leff, D. R. (2020). Systematic review of combined functional near-infrared spectroscopy and transcranial direct-current stimulation studies. Neurophotonics 7:020901. doi: 10.1117/1. NPh.7.2.020901

Peng, W. W., Tang, Z. Y., Zhang, F. R., Li, H., Kong, Y. Z., Iannetti, G. D., et al. (2019). Neurobiological mechanisms of TENS-induced analgesia. Neuroimage 195, 396-408. doi: 10.1016/j.neuroimage.2019. 03.077

Peters, S., Lim, S. B., Louie, D. R., Yang, C. L., and Eng, J. J. (2020). Passive, yet not inactive: robotic exoskeleton walking increases cortical activation dependent on task. J. Neuroeng. Rehabil. 17:107. doi: 10.1186/s12984-02000739-6

Pinti, P., Scholkmann, F., Hamilton, A., Burgess, P., and Tachtsidis, I. (2019). Current status and issues regarding pre-processing of fNIRS neuroimaging data: an investigation of diverse signal filtering methods within a general linear model framework. Front. Hum. Neurosci. 12:505. doi: 10.3389/fnhum.2018. 00505

Quaresima, V., and Ferrari, M. (2019). A mini-review on functional near-infrared spectroscopy (fNIRS): where do we stand, and where should we go? Photonics Basel 6:87. doi: 10.3390/photonics6030087

Rojas, R. F., Liao, M. Y., Romero, J., Huang, X., and Ou, K. L. (2019). Cortical network response to acupuncture and the effect of the hegu point: an fNIRS study. Sensors Basel 19:394. doi: 10.3390/s19020394

Rubinov, M., and Sporns, O. (2010). Complex network measures of brain connectivity: Uses and interpretations. Neuroimage 52, 1059-1069. doi: 10. 1016/j.neuroimage.2009.10.003

Scholkmann, F., Kleiser, S., Metz, A. J., Zimmermann, R., Pavia, J. M., Wolf, U., et al. (2014). A review on continuous wave functional near-infrared spectroscopy and imaging instrumentation and methodology. Neuroimage 85, 6-27. doi: 10.1016/j.neuroimage.2013.05.004

Shmuel, A., Yacoub, E., Pfeuffer, J., Van de Moortele, P. F., Adriany, G., Hu, X. P., et al. (2002). Sustained negative BOLD, blood flow and oxygen consumption response and its coupling to the positive response in the human brain. Neuron 36, 1195-1210. doi: 10.1016/S0896-6273(02)01061-9

Soltanlou, M., Sitnikova, M. A., Nuerk, H. C., and Dresler, T. (2018). Applications of functional near-infrared spectroscopy (fNIRS) in studying cognitive development: the case of mathematics and language. Front. Psychol. 9:277. doi: 10.3389/fpsyg.2018.00277

Stefanovic, B., Warnking, J. M., and Pike, G. B. (2004). Hemodynamic and metabolic responses to neuronal inhibition. Neuroimage 22, 771-778. doi: 10. 1016/j.neuroimage.2004.01.036

Strangman, G., Culver, J. P., Thompson, J. H., and Boas, D. A. (2002). A quantitative comparison of simultaneous BOLD fMRI and NIRS recordings during functional brain activation. Neuroimage 17, 719-731. doi: 10.1006/nimg. 2002.1227

Sun, R. R., He, Z. X., Ma, P. H., Yin, S., Yin, T., Liu, X. Y., et al. (2020). The participation of basolateral amygdala in the efficacy of acupuncture with deqi treating for functional dyspepsia. Brain Imaging Behav. 15, 216-230. doi: 10. 1007/s11682-019-00249-7

Tabar, Y. R., and Halici, U. (2017). Brain computer interfaces for silent speech. Eur. Rev. 25, 208-230. doi: 10.1017/S1062798716000569 
Takahashi, T. (2011). Mechanism of acupuncture on neuromodulation in the gut-a review. Neuromodulation 14, 8-12. doi: 10.1111/j.1525-1403.2010. 00295.x

Takamoto, K., Hori, E., Urakawa, S., Sakai, S., Ishikawa, A., Kohno, S., et al. (2010). Cerebral hemodynamic responses induced by specific acupuncture sensations during needling at trigger points: a near-infrared spectroscopic study. Brain Topogr. 23, 279-291. doi: 10.1007/s10548-010-0148-8

Tsuzuki, D., Jurcak, V., Singh, A. K., Okamoto, M., Watanabe, E., and Dan, I. (2007). Virtual spatial registration of stand-alone fNIRS data to MNI space. Neuroimage 34, 1506-1518. doi: 10.1016/j.neuroimage.2006.10.043

van den Heuvel, M. P., and Pol, H. E. H. (2010). Exploring the brain network: A review on resting-state fMRI functional connectivity. Eur. Neuropsychopharm. 20, 519-534. doi: 10.1016/j.euroneuro.2010.03.008

van den Heuvel, M. P., Scholtens, L. H., de Lange, S. C., Pijnenburg, R., Cahn, W., van Haren, N. E. M., et al. (2019). Evolutionary modifications in human brain connectivity associated with schizophrenia. Brain 142, 3991-4002. doi: 10.1093/brain/awz330

Volz, M. S., Suarez-Contreras, V., Portilla, A. L. S., Illigens, B., Bermpohl, F., and Fregni, F. (2015). Movement observation-induced modulation of pain perception and motor cortex excitability. Clin. Neurophysiol. 126, 1204-1211. doi: 10.1016/j.clinph.2014.09.022

Wang, J. H., Wang, X. D., Xia, M. R., Liao, X. H., Evans, A., and He, Y. (2015). GRETNA: a graph theoretical network analysis toolbox for imaging connectomics. Front. Hum. Neurosci. 9:386. doi: 10.3389/fnhum.2015. 00386

Wang, Q., Zhu, G. P., Yi, L., Cui, X. X., Wang, H., Wei, R. Y., et al. (2020). A review of functional near-infrared spectroscopy studies of motor and cognitive function in preterm infants. Neurosci. Bull. 36, 321-329. doi: 10.1007/s12264019-00441-1

Wang, S. S., Itthipuripat, S., and Ku, Y. X. (2019). Electrical stimulation over human posterior parietal cortex selectively enhances the capacity of visual short-term memory. J. Neurosci. 39, 528-536. doi: 10.1523/Jneurosci.1959-18.2018

Wang, Z. Q., Liang, P. P., Zhao, Z. L., Han, Y., Song, H. Q., Xu, J. Y., et al. (2014). Acupuncture modulates resting state hippocampal functional connectivity in Alzheimer disease. PLoS One 9:91160. doi: 10.1371/journal.pone.0091160

Xia, M. R., Wang, J. H., and He, Y. (2013). BrainNet viewer: a network visualization tool for human brain connectomics. PLoS One 8:68910. doi: 10.1371/journal. pone.0068910

Yamada, T., Umeyama, S., and Matsuda, K. (2012). Separation of fNIRS signals into functional and systemic components based on differences in hemodynamic modalities. PLoS One 7:50271. doi: 10.1371/journal.pone.0050271

Yang, X. Y., Shi, G. X., Li, Q. Q., Zhang, Z. H., Xu, Q., and Liu, C. Z. (2013). Characterization of Deqi sensation and acupuncture effect. Evid. Based Complement. Alternat. Med. 2013:319734. doi: 10.1155/2013/ 319734

Yoo, S. S., Teh, E. K., Blinder, R. A., and Jolesz, F. A. (2004). Modulation of cerebellar activities by acupuncture stimulation: evidence from fMRI study. Neuroimage 22, 932-940. doi: 10.1016/j.neuroimage.2004.02.017

Yu, D. T. W., Jones, A. Y. M., and Pang, M. Y. C. (2012). Development and validation of the Chinese version of the Massachusetts General Hospital Acupuncture Sensation Scale: an exploratory and methodological study. Acupunct. Med. 30, 214-221. doi: 10.1136/acupmed-2012-010145

Yu, H. T., Li, X., Lei, X. Y., and Wang, J. (2019). Modulation effect of acupuncture on functional brain networks and classification of its manipulation with EEG signals. IEEE Trans. Neural Syst. Rehabil. Eng. 27, 1973-1984. doi: 10.1109/ Tnsre.2019.2939655

Yu, H. T., Liu, J., Cai, L. H., Wang, J., Cao, Y. B., and Hao, C. Q. (2017). Functional brain networks in healthy subjects under acupuncture stimulation: An EEG study based on nonlinear synchronization likelihood analysis. Physica A 468, 566-577. doi: 10.1016/j.physa.2016.10.068

Yu, H. T., Wu, X. Y., Cai, L. H., Deng, B., and Wang, J. (2018). Modulation of spectral power and functional connectivity in human brain by acupuncture stimulation. IEEE Trans. Neural Syst. Rehabil. Eng. 26, 977-986. doi: 10.1109/ Tnsre.2018.2828143

Yucel, M. A., Selb, J., Aasted, C. M., Lin, P. Y., Borsook, D., Becerra, L., et al. (2016). Mayer waves reduce the accuracy of estimated hemodynamic response functions in functional near-infrared spectroscopy. Biomed. Opt. Express 7, 3078-3088. doi: 10.1364/Boe.7.003078

Zeng, L. L., Shen, H., Liu, L., Wang, L. B., Li, B. J., Fang, P., et al. (2012). Identifying major depression using whole-brain functional connectivity: a multivariate pattern analysis. Brain 135, 1498-1507. doi: 10.1093/brain/aws059

Zhang, H., Duan, L. A., Zhang, Y. J., Lu, C. M., Liu, H. L., and Zhu, C. Z. (2011). Test-retest assessment of independent component analysis-derived restingstate functional connectivity based on functional near-infrared spectroscopy. Neuroimage 55, 607-615. doi: 10.1016/j.neuroimage.2010.12.007

Zhang, Y., Li, K., Ren, Y., Cui, F., Xie, Z., Shin, J. Y., et al. (2014). Acupuncture modulates the functional connectivity of the default mode network in stroke patients. Evid. Based Complement. Alternat. Med. 2014:765413. doi: 10.1155/ 2014/765413

Zhang, Z. J., Wang, X. M., and McAlonan, G. M. (2012). Neural acupuncture unit: a new concept for interpreting effects and mechanisms of acupuncture. Evid. Based Complement. Alternat. Med. 2012:429412. doi: 10.1155/2012/ 429412

Zheng, W. M., Su, Z. Z., Liu, X. Y., Zhang, H., Han, Y., Song, H. Q., et al. (2018). Modulation of functional activity and connectivity by acupuncture in patients with Alzheimer disease as measured by resting-state fMRI. PLoS One 13:196933. doi: 10.1371/journal.pone.0196933

Zhong, C. G., Bai, L. J., Dai, R. W., Xue, T., Wang, H., Feng, Y. Y., et al. (2012). Modulatory effects of acupuncture on resting-state networks: A functional MRI study combining independent component analysis and multivariate granger causality analysis. J. Magn. Reson. Imaging 35, 572-581. doi: 10.1002/jmri. 22887

Zhu, H. L., Xu, J., Li, J. X., Peng, H. J., Cai, T. T., Li, X. G., et al. (2017). Decreased functional connectivity and disrupted neural network in the prefrontal cortex of affective disorders: A resting-state fNIRS study. J. Affect. Disorders 221, 132-144.

Zuo, X. N., Di Martino, A., Kelly, C., Shehzad, Z. E., Gee, D. G., Klein, D. F., et al. (2010). The oscillating brain: complex and reliable. Neuroimage 49, 1432-1445. doi: 10.1016/j.neuroimage.2009.09.037

Conflict of Interest: The authors declare that the research was conducted in the absence of any commercial or financial relationships that could be construed as a potential conflict of interest.

Publisher's Note: All claims expressed in this article are solely those of the authors and do not necessarily represent those of their affiliated organizations, or those of the publisher, the editors and the reviewers. Any product that may be evaluated in this article, or claim that may be made by its manufacturer, is not guaranteed or endorsed by the publisher.

Copyright (c) 2021 Si, Xiang, Zhang, Li, Zhang and Ming. This is an open-access article distributed under the terms of the Creative Commons Attribution License (CC BY). The use, distribution or reproduction in other forums is permitted, provided the original author(s) and the copyright owner(s) are credited and that the original publication in this journal is cited, in accordance with accepted academic practice. No use, distribution or reproduction is permitted which does not comply with these terms. 